\title{
Submillimeter Site Testing at Dome C, Antarctica
}

\author{
Paolo G. Calisse ${ }^{\mathrm{A}, \mathrm{D}}$, Michael C. B. Ashley ${ }^{\mathrm{A}}$, Michael G. Burton ${ }^{\mathrm{A}}$, Michael A. Phillips ${ }^{\mathrm{A}}$, \\ John W. V. Storey ${ }^{\mathrm{A}}$, Simon J. E. Radford ${ }^{\mathrm{B}}$, and Jeffrey B. Peterson ${ }^{\mathrm{C}}$ \\ ${ }^{\text {A }}$ School of Physics, University of New South Wales, Sydney NSW 2052, Australia \\ B National Radio Astronomy Observatory, Tucson AZ 85721-0655, USA \\ ${ }^{\mathrm{C}}$ Department of Physics, Carnegie Mellon University, Pittsburgh PA 15213-3890, USA \\ D E-mail: pcalisse@phys.unsw.edu.au
}

Received 2003 March 17, accepted 2004 February 6

\begin{abstract}
We have developed a $350 \mu \mathrm{m}$ radiometer to perform automated site testing in remote regions of Antarctica. In summer 2000-2001 the instrument operated at Concordia, a new station under construction at Dome $\mathrm{C}$ on the Antarctic Plateau. We present the results, and compare them with the atmospheric opacity measured at the South Pole in the same five-week period. During these five weeks, observing conditions at Dome $\mathrm{C}$ were, on average, substantially better than those at the South Pole.
\end{abstract}

Keywords: atmospheric effects — instrumentation: miscellaneous — site testing — submillimeter

\section{Introduction}

For many years (Burova et al. 1986; Smythe \& Jackson 1977; Townes \& Melnick 1990; Storey \& Hyland 1992) the Antarctic Plateau has been considered one of the most promising sites in the world for submillimetre astronomy because its high altitude and extremely dry, stable, and cold atmosphere. After the first summer measurements (Dragovan et al. 1990; Pajot et al. 1989) the Antarctic Submillimetre Telescope and Remote Observatory (AST/RO) was installed at the South Pole (Stark et al. 2001). However, while the South Pole has indeed proven to be an exceptionally good site (Chamberlin 2001; Chamberlin, Lane, \& Stark 1997), other sites on the Antarctic Plateau could provide still better transparencies and lower atmospheric emission and fluctuations at this and at other wavelength regions, as they feature an higher elevation and are located on the top of a dome where katabatic (gravity-driven) winds, and consequently atmospheric turbulence, are expected to be much lower. Among these sites are Dome $\mathrm{C}\left(75^{\circ} 05^{\prime} \mathrm{S}, 123^{\circ} 06^{\prime} \mathrm{E}\right.$, alt. $3260 \mathrm{~m}$ asl), Dome Argus $\left(81^{\circ} 00^{\prime} \mathrm{S}, 77^{\circ} 00^{\prime} \mathrm{E}\right.$, alt. $4000 \mathrm{~m}$ asl), and Dome Fuji $\left(77^{\circ} 19^{\prime} \mathrm{S}, 39^{\circ} 42^{\prime} \mathrm{E}, 3800 \mathrm{~m}\right.$ asl). Only at Dome $\mathrm{C}$ is a permanent manned station under construction (Godon \& Cucinotta 2000; Candidi \& Lori 2003). Preliminary site testing in the millimetre wavelength region at Dome C carried out in 1995 (Valenziano \& Dall'Oglio 1999) resulted in stability measurements and encouraging upper limits for the atmospheric precipitable water vapour (PWV).

In this paper, we present opacity data in the $350 \mu \mathrm{m}$ atmospheric window, obtained during a site testing campaign carried out in summer 2000-2001 at Dome C. We compare these data with results obtained at the South Pole with a similar instrument over the same period. A direct comparison of the performance of the two instruments was also made by acquiring further data simultaneously at the South Pole with both instruments.

\section{Concordia Station}

Dome $\mathrm{C}$ is the site of a new permanent station, Concordia, currently under construction by Italy and France. The station sits on one of the high points of the Plateau, hence the geographical name.

Winds on the Antarctic Plateau are frequently katabatic and are created by colder air masses sliding down the small, but non-zero average slope of the Plateau (Mastrantonio et al. 1999; Schwerdtfeger 1984). For this reason, at sites such as the South Pole that are not at domes, a slow but almost constant wind is experienced which increases the air turbulence in the atmospheric boundary layer.

The meteorological records from the American Amundsen-Scott station at the South Pole and from the Russian Vostok Station, both operating since the International Geophysical Year (1957), show an average wind about twice that at Dome C. In particular, during the winter season a weather station installed at Concordia Station reported very low $\left(<0.5 \mathrm{~m} \mathrm{~s}^{-1}\right)$ wind speed for most of the period of complete darkness (data courtesy ENEA-PNRA 2001). Comparative data on the three stations are shown in Table 1.

Dome C summer camp is currently operating from early December to the first week of February each year, while the Concordia winter station is expected to be open by winter 2005 . Heavy equipment is moved by ground traverse to Dome C from the station Dumont d'Urville, $1200 \mathrm{~km}$ distant, and located on the sector of the Antarctic coast facing Tasmania. Each traverse, of which there are 
Table 1. Characteristics of the three permanent stations located on the Antarctic Plateau

\begin{tabular}{|c|c|c|c|}
\hline & Vostok & Concordia & $\begin{array}{l}\text { Amundsen- } \\
\text { Scott }\end{array}$ \\
\hline Latitude & $78^{\circ} 30^{\prime} \mathrm{S}$ & $75^{\circ} 06^{\prime} \mathrm{S}$ & $90^{\circ} \mathrm{S}$ \\
\hline Longitude & $123^{\circ} 48^{\prime} \mathrm{E}$ & $123^{\circ} 06^{\prime} \mathrm{E}$ & - \\
\hline Elevation [m] & 3488 & 3260 & 2830 \\
\hline $\begin{array}{l}\text { Average } \\
\text { pressure }[\mathrm{hPa}]\end{array}$ & 624 & 644 & 680 \\
\hline $\begin{array}{l}\text { Minimum } \\
\text { temperature }\left[{ }^{\circ} \mathrm{C}\right]\end{array}$ & -89 & -84 & -82 \\
\hline $\begin{array}{l}\text { Average } \\
\text { temperature }\left[{ }^{\circ} \mathrm{C}\right]\end{array}$ & -58 & -51 & -49 \\
\hline $\begin{array}{l}\text { Average wind } \\
\text { speed }\left[\mathrm{m} \mathrm{s}^{-1}\right]\end{array}$ & 5.0 & 2.8 & 5.8 \\
\hline Activity period & $\begin{array}{c}\text { Permanently } \\
\text { since } 1957\end{array}$ & $\begin{array}{l}\text { Summers } \\
\text { since } 1997 \\
\text { Winters from } \\
2005 \text { (expected) }\end{array}$ & $\begin{array}{c}\text { Permanently } \\
\text { since } 1957\end{array}$ \\
\hline
\end{tabular}

currently three each summer, carries about 130 metric tonnes of payload. Items carried to Dome $\mathrm{C}$ are therefore not subject to the size or weight limits imposed by air transport. This could represent an important benefit for the transport of oversized scientific equipment.

A $4200 \mathrm{~m} \times 100 \mathrm{~m}$ compact ice runway is available for large, ski-equipped aircraft operations. For personnel and light items an air link is available on ski-equipped aircraft that in summer connect Dome $\mathrm{C}$ to the three coastal stations of Terra Nova Bay (Italy) and McMurdo (USA), both located on the Ross Sea, and Dumont d'Urville (France).

Geostationary communication satellites are permanently visible at the latitude of the station and can provide broadband communication 24 hours a day. Further discussion of the potential of Dome C can be found in Dopita et al. (1996) and Storey et al. (2003).

Since winter 2001 a cloud-cover monitor developed at the University of New South Wales (UNSW) has been automatically operating at Dome C. At present, the summer camp at Dome $\mathrm{C}$ can supply only limited logistic support to science, but instrumentation for site testing and astronomy has been or is planned to be deployed at Dome $\mathrm{C}$ in the next few years, in order to explore the expected excellent capabilities in the submillimetre and infrared region of the electromagnetic spectrum. The AASTINO, a multiwavelength, winterover, automated module for site testing built by UNSW, has been moved at the site during summer 2002-2003.

\section{Instrument Setup}

The SUMMIT (or SUb-MilliMeter Tipper) device is developed from a series of four essentially identical radiometers built by the National Radio Astronomy Observatory (NRAO) and Carnegie Mellon University (CMU). The original tipper design, sometimes known as the 'NRAO tau-meter', was created in order to facilitate a direct comparison of sky opacity in the $350 \mu \mathrm{m}$ submillimetre atmospheric window at different sites (Radford \& Holdaway 1998). The first three units have already been deployed to where large submillimetre facilities have been built, or are under planning or construction: Llano de Chajnantor (Chile), Mauna Kea (Hawaii), and the South Pole (Antarctica). Results to date from these tippers are summarised by Radford (2002). The fourth unit, SUMMIT, has been modified at UNSW to reduce electrical power requirements, thus allowing operation in remote, unoccupied sites in inner Antarctica.

All four tippers were designed to operate with ambient temperature detectors, to reduce costs and maintenance requirements. Radiation from the sky is collected by a $15 \mathrm{~cm}$ diameter, $45^{\circ}$ off-axis paraboloid mirror, giving an instrument field of view of approximately $6^{\circ}$. The mirror can rotate through $360^{\circ}$ to scan the sky from $0^{\circ}$ to $90^{\circ}$ of elevation and, in addition, to look at two black bodies maintained at different temperatures. These two blackbodies form the basis of the instrument's absolute calibration.

The beam from the sky (or from the blackbodies) is directed through a chopper, and then to a blocking filter and to an $837 \mathrm{GHz}$ mesh filter with a FWHM bandwidth of $108 \mathrm{GHz}$. All four instruments use mesh filters from the same manufacturer (QMC Instruments) and are as nearly identical as possible. A Winston cone is used to define the $A \Omega$ value of the system and to couple the radiation to the detector.

The two calibration blackbodies are identical in construction. Each is machined from Eccosorb MF-110, a magnetically loaded epoxy. The front surface is corrugated to allow multiple reflections, improving the emissivity, and is protected by a double layer of Zytex, a very thin randomfibre Teflon-based material, thermally insulating the black bodies and decreasing thermal inhomogeneities. Typically, the temperature difference between the two black bodies in Antarctica ranges from 25 to $40^{\circ} \mathrm{C}$. The temperature of each blackbody is measured by a platinum RTD, giving an absolute temperature calibration of $\pm 0.1^{\circ} \mathrm{C}$. The temperature of the corners of each blackbody was also continuously monitored with a set of Dallas DS1820 sensors to check for any temperature gradients across the face of the blackbody.

Although it is important to keep SUMMIT compatible with the other $350 \mu \mathrm{m}$ tau-meters described above, it was necessary to make several modifications to the original design to reduce electrical power consumption, for deployment at remote sites where only small amounts of electrical power will be available.

In the original tau-meter design, the two blackbodies were electrically heated. In SUMMIT, this electrical heating is dispensed with completely. One of the black bodies, described in the following as the 'warm' one, is maintained in thermal contact with a large copper heat sink. At the South Pole, this heat sink is exposed to the room air of the AASTO (Storey 1998; Storey, Ashley, \& Burton 1996), while at Dome C it was enclosed in a temperature 
controlled box that also housed the electronics and computer.

The other blackbody, the 'cold' one, is attached directly to the outer case of SUMMIT, thus placing it in thermal contact with the outdoor environment. While this has proven to be a successful approach in most situations, we found that the bright sunshine at Dome $\mathrm{C}$ in mid-summer could cause excessive heating of the 'cold' blackbody. After a few days of operations at Dome C, a sunshield was therefore installed around the instrument to protect it from direct sunlight (the data presented in this paper were all taken after this modification).

SUMMIT also uses a different pyroelectric detector to the original tau-meters. The new detector is a Barnes DLATGS 2-1162-8-01 with a specified NEP of $2.1 \times 10^{-10} \mathrm{~W} \mathrm{~Hz}^{1 / 2}$ at $10 \mathrm{~Hz}$. This detector also has a faster response than the original devices, allowing the chopping frequency to be increased to $10.7 \mathrm{~Hz}$ (from the original $0.75 \mathrm{~Hz}$ ) and thereby reducing $1 / f$ noise.

The SUMMIT chopper is driven by a low-power brushless DC motor of the type used in the NISM and MISM infrared sky monitors (Storey et al. 1999). A phaselocked-loop drive keeps the motor running at precisely the correct frequency, allowing a relatively narrow-band filter ( $Q=10)$ to be used in the signal chain to improve dynamic range. After several stages of amplification the signal is digitised, processed in a software implementation of a digital lock-in, and then stored on a PC/104 solid-state disk, along with the position of the mirror and several other environmental and instrument parameters. The system is controlled by software written within the ERIC programming environment (Ashley, Brooks, \& Lloyd 1996). All the instrument components, except those in the optical path, are protected by a thick layer of thermal insulator to prevent thermal losses and to maintain the sensitive devices within their working temperature range without the use of electrical heaters. The average power consumption of the instrument is less than $4 \mathrm{~W}$.

The instrument is protected from the atmosphere by a radome. During the summer 2001-2002 campaign at Dome C (and, later, at the South Pole), the radome consisted of a thin layer of woven Gore-Tex. The transparency of this material is affected by the incident angle and by several other factors (Afsar, Tkachov, \& Wells 1996; Afsar, Chi, \& Tkachov 1995). We therefore made a careful measurement of the overall transparency in our laboratory by observing a calibrated source with SUMMIT, finding a transmission of $\sim 77 \%$ at $\sim 300 \mathrm{~K}$.

We stress that the modifications made to SUMMIT have left unchanged all parts of the tipper that determine its calibration - namely, the Winston cone, filter, blocking filter, blackbody sources, and optics. This ensures that results from SUMMIT should be directly comparable to those from the other three instruments. In addition, we have made a month-long side-by-side comparison of SUMMIT and one of the original tau-meters, as described in Section 7.

\section{The Antarctic Submillimetre Site Testing Program}

SUMMIT was deployed at Dome C on 2000 December 4 . The instrument was fully operational from 2000 December 8 until 2001 January 26, at which time it was relocated to the South Pole, and installed on the top of the AASTO (Storey et al. 1996). The AASTO provided electrical power and the heat required to maintain the warmer black body at the operating temperature.

At the end of 2001 February the two instruments were tested side-by-side at the South Pole. For the first $48 \mathrm{~h}$ (50 data points), there was extremely tight correspondence, the offset being $\tau_{\text {SUMMIT }}-\tau_{\mathrm{NRAO} / \mathrm{CMU}}=0.1 \pm 0.1$, where the two $\tau$ values represent respectively the average of the SUMMIT and of the NRAO/CMU tipper opacity measured during the whole time interval. This offset, though very small, is in the direction that would make the difference between Dome C and the South Pole even greater than presented here.

After that, SUMMIT began to show increasingly pessimistic values of the zenith atmospheric opacity $\tau_{0}$. This was later traced to improper sealing, allowing ice to accumulate on the inside of the window (we do not believe that there is any such problem with SUMMIT's Dome C data, as the instrument set-up at Dome $\mathrm{C}$ did not expose it to inside-air humidity).

\section{Calibration and Data Analysis}

The observing strategy was adjusted several times during the first few weeks of the Dome $\mathrm{C}$ campaign, both to improve the performance of the system and to correct minor problems. A final tuning of the hardware and of the automatic data acquisition script was achieved by 2000 December 21.

An average value of the overall responsivity, $G$, of the instrument has been obtained using the whole dataset from that date until the end of the campaign, on 2001 January 26 , by periodic measurements of the signal from cold and warm black bodies and their temperatures, Equation (1):

$$
G=\frac{S_{\text {cold }}-S_{\text {warm }}}{T_{\text {warm }}-T_{\text {cold }}}
$$

where $S_{\text {warm }}$ and $S_{\text {cold }}$ are the output of the detector amplifier chain [V] when the detector is observing the warm and cold blackbody respectively, and $T_{\text {warm }}$ and $T_{\text {cold }}$ are the absolute temperatures of the blackbodies. Data up to December 21, although not biased, were too noisy to improve the evaluation of the average responsivity. There is no evidence that the responsivity varies either with time or with detector temperature.

The responsivity has been monitored during the whole of the campaign, to check for possible system malfunctioning or change in performance. A best fit gives a value for the responsivity $G=0.0073 \pm 0.0009 \mathrm{~V} \mathrm{~K}^{-1}$. This value has been used in all further data analysis.

The first dataset from, December 8-11, was manually acquired, from December 11-20 an automated script was used to acquire the sky signal at seven different airmasses 
$(1,1.5,2,2.5,3,3.5,4)$. Each data point was integrated for $10 \mathrm{~s}$, in a way similar to the original observing strategies used by the other tippers.

After December 21 the script running the instrument was modified to sample the signal four consecutive times at each elevation for $\sim 10 \mathrm{~s}$ at $3.6^{\circ}$ elevation steps, starting from $10^{\circ}$ away from the zenith on one side, through the zenith, then down to the horizon on the opposite side. This observing strategy was demonstrated to be very effective, and had been maintained with little change until 2001 January 26, when the impending station closure forced the removal of the instrument.

In the first period (December 8-11) the number of valid opacity values obtained was $\sim 1.9 \mathrm{~h}^{-1}$. In the second (December 11-21) $8.6 \mathrm{~h}^{-1}$, then $2.9 \mathrm{~h}^{-1}$ until the end of the campaign, for a total amount of $\sim 4600$ skydips in a 49-day campaign.

The lock-in integration time was $\sim 9.3 \mathrm{~s}$, corresponding to 100 chop cycles at $10.7 \mathrm{~Hz}$. About 40 of these $9.3 \mathrm{~s}$ integrations were made at each elevation. An ADC sampled the waveform at the maximum and the minimum at each cycle. The lock-in output was the average of the difference between these maxima and minima. Noisy samples (samples featuring high fluctuations from cycle to cycle), mainly related to electronic or mechanical noise due to human activities in the vicinity, were rejected. The total number of rejected data points was less than 1/3000 of the total number of samples used for this analysis.

The chopper blade is made from polished aluminium. The instrument is switching between it and the external radiation. When the chopper was closed, the detector and the concentrator formed a closed, reflecting cavity, so that the detector was measuring in part the radiation emitted by itself, and in part scattered radiation from the instrument enclosure.

The reference temperature $T_{\text {ref }}$ during each skydip was obtained through Equation (2):

$$
S_{\mathrm{bb}}=G\left(T_{\mathrm{ref}}-T_{\mathrm{bb}}\right)
$$

where $T_{\mathrm{bb}}$ is the temperature of one of the black bodies and $S_{\mathrm{bb}}$ the signal detected when looking at that blackbody.

According to the isothermal slab atmospheric model, the atmosphere can be described as a single layer at temperature $T_{\text {atm }}$ and zenith opacity $\tau_{0}$. Two different methods have been used to obtain $\tau_{0}$ and $T_{\mathrm{atm}}$.

The first method makes use of a non-linear fit to data obtained at several different airmasses. Under these conditions Equation (3) holds

$$
\begin{aligned}
S(X)= & G\left\{\theta_{\text {win }} T_{\text {atm }}\left[1-\exp \left(-X \tau_{0}\right)\right]\right. \\
& \left.-T_{\text {ref }}+T_{\text {win }}\left(1-\theta_{\text {win }}\right)\right\}
\end{aligned}
$$

where $S(X)$ is the signal at the output of the amplifier chain, and $X(z) \equiv \cos ^{-1}(z)$ the airmass number at zenith distance $z . \theta_{\text {win }}$ and $T_{\text {win }}$ are, respectively, the transparency and the temperature of the Gore-Tex radome protecting the instrument. $T_{\text {atm }}$ and $\tau_{0}$ are the two unknown parameters derived by applying a Levenberg-Marquardt (LM) fitting

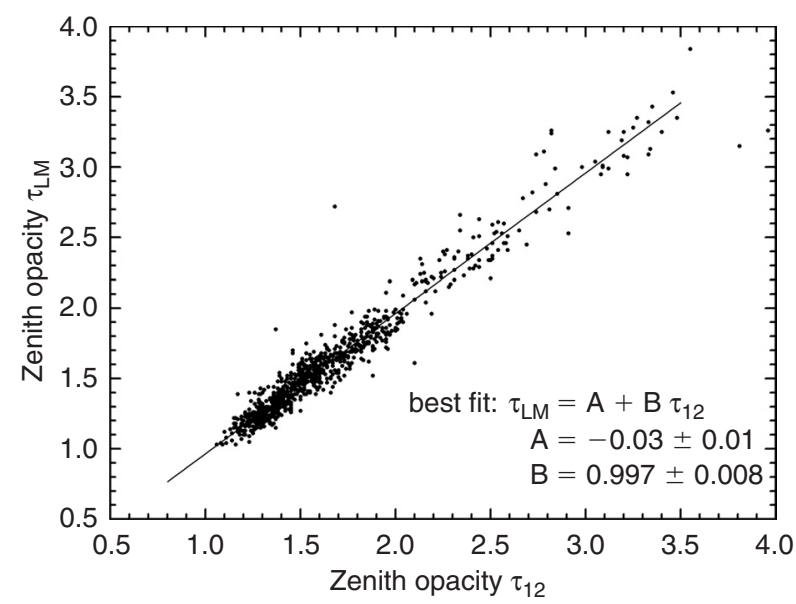

Figure 1 Correlation between opacity obtained from data at 1 and 2 airmasses, and that obtained from a non-linear fit of data acquired at 24 different evenly spaced elevations.

routine to the complete skydip of 24 points. Data at zenith distances larger than $75^{\circ}$ have not been used in the fit to avoid contamination by spillover from the ground, and because, given the large field of view, the airmass number is not well defined at low elevation.

We included in Equation (3) the effect of the Gore-Tex radome protecting the instrument. As shown in another paper (Calisse 2004), the radome introduces a non-linear effect into the results and must be taken in account. This effect is shown as the last additive term of Equation (3).

The second method makes use of data at 1 and 2 airmasses only. These two points are taken from the same dataset used in the LM method, or from independent skydips. From Equation (3), computed at 1 and 2 airmasses, one obtains Equations (4) and (5)

$$
\begin{aligned}
\exp \left(-\tau_{0}\right) & =\frac{T_{2}-T_{1}}{T_{1}-T_{\text {win }}\left(1-\theta_{\text {win }}\right)+T_{\text {ref }}} \\
T_{\text {atm }} & =\frac{T_{1}-T_{\text {win }}\left(1-\theta_{\text {win }}\right)+T_{\text {ref }}}{\theta_{\text {win }}\left[1-\exp \left(-t_{0}\right)\right]}
\end{aligned}
$$

where $T_{n}=S_{n} / G$ for the signals at 1 and 2 airmasses expressed in units of temperature. This strategy makes more efficient use of the observing time and also reduces the overhead time. Once again it is necessary to properly include the effect of the radome (Calisse 2004).

Figure 1 shows that the values obtained using the two different methods are extremely well correlated and that the dispersion of the two datasets is about the same. However, in order to maintain full compatibility with the measurements obtained by the other submillimetre tippers, we acquired data over the whole airmass range during the entire Dome $\mathrm{C}$ campaign.

\section{Weather Conditions}

Weather data measured from an automatic 'Vaisala AW11 Aviation Weather Reporter' system, used at Dome C for 

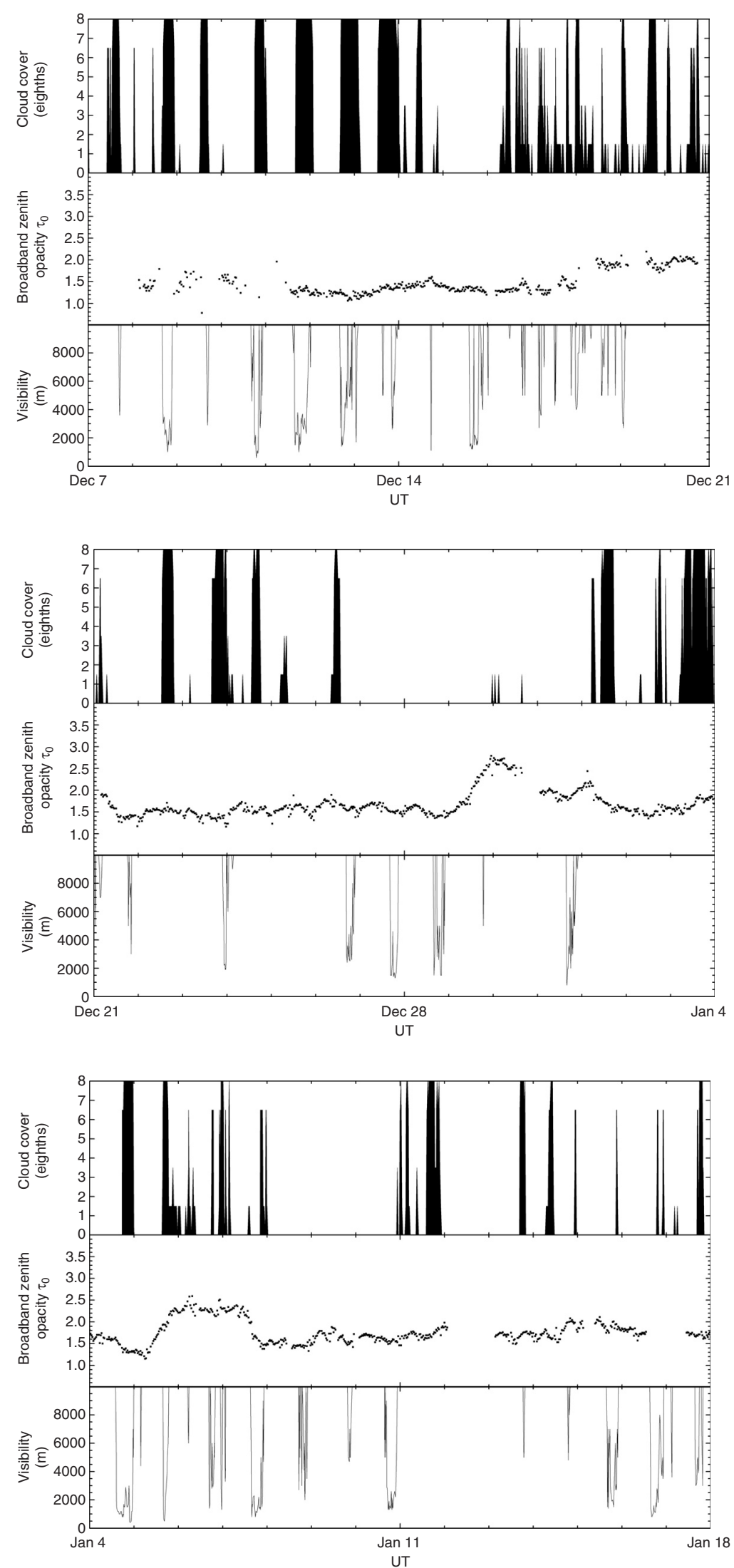

Figure 2 Data series from the Dome C summer 2000-2001 site testing campaign shown with relevant parameters acquired from a Vaisala AW11 Aviation Weather Reporter operating at the station. The visibility is defined as the horizontal visibility; $350 \mu \mathrm{m}$ opacity data come from the present work; cloud cover (from METAR every $30 \mathrm{~min}$, from SCITAR when a sudden change in conditions is monitored) messages have been converted in numerical quantities, see text. 


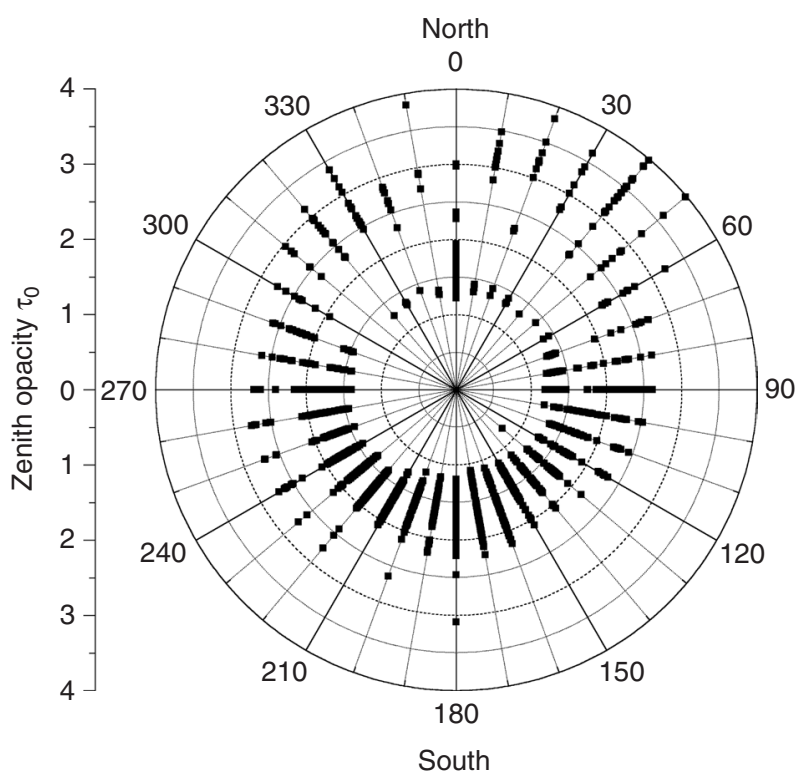

Figure 3 Atmospheric opacity as a function of the incoming wind direction at Dome C. Opacity and winds values averaged in $0.5 \mathrm{~h}$ intervals. When no wind was detected the corresponding opacity was assigned to $0^{\circ}$.

air traffic support, have been used to check for a possible correlation between cloud cover and submillimetre opacity. Standard AW11 measurements include cloud layer height and coverage, visibility, air pressure, temperature, dew point, and wind speed and direction. The record is shown in Figure 2, where the visibility represents the horizontal visibility (defined as the maximum distance at which an object can be distinguished on a horizontal line of sight), and the cloud-covered fraction (measured in 'eighths' of sky covered). Clear sky is $0 / 8$; few clouds $1 / 8-2 / 8$, scattered clouds $3 / 8-4 / 8$, broken clouds $5 / 8-7 / 8$, and overcast 8/8 (AW11 data courtesy of ENEA-PNRA).

Perhaps surprisingly, there is no evidence of a correlation between submillimetre atmospheric opacity and cloud conditions. These clouds consist mainly of tiny ice crystals, which are apparently unable to scatter or absorb submillimetre radiation to any significant extent.

Several other meteorological parameters have also been monitored during this period. The wind speed ranged from 0 to $7.7 \mathrm{~m} \mathrm{~s}^{-1}$ with an average value of $3.2 \mathrm{~m} \mathrm{~s}^{-1}$, and the ambient temperature from -41 to $-19^{\circ} \mathrm{C}$ with an average value of $-31.2^{\circ} \mathrm{C}$.

Possible correlations between the zenith opacity and other meteorological parameters have been explored. While there is no evidence of a clear relation between opacity data and pressure or ground temperature, a strong correlation has been observed with wind direction. When the wind is flowing from the highest, driest regions of the Antarctic Plateau (located to the south-west of Dome C) wind speed and sky opacity tend towards lower values. When the air is coming from the direction of the coastal regions, the air is warmer and therefore may contain more moisture, leading to a higher opacity. These results are shown in Figure 3. Similar characteristics have

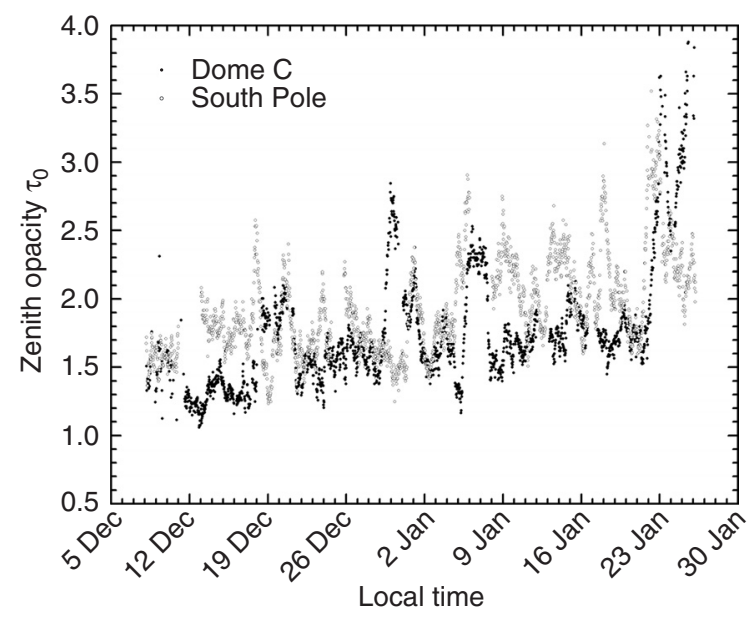

Figure 4 Opacity at Dome C and the South Pole in summer 20002001. Dome C values are seen to be significantly lower and more stable for most of the time.

been found at the South Pole in the $492 \mathrm{GHz}$ atmospheric window (Chamberlin et al. 1997). At that site the driest atmosphere and the highest transparency are associated with katabatic winds flowing from the highest sites of the Plateau.

\section{Discussion}

The opacity values obtained at Dome C, compared to values obtained simultaneously at the South Pole with the similar tipper, are shown in Figure 4.

The cumulative distribution of $\tau_{0}$ is reported in Figure 5 where it is evident a significant difference in the average value. Moreover, it is evident from Figure 4 that the conditions at Dome $\mathrm{C}$ are more stable. Quartile opacity values are given in Table 2 .

Although five and half weeks of simultaneous operation of the almost identical submillimetre tippers at the South Pole and Dome C do not by any means represent a complete statistical sample, there is evidence that transparency conditions in the submillimetre wavelength region at Dome $\mathrm{C}$ may be substantially better, with an average difference in sky opacity greater than 0.2 between the two sites in summer.

The slight difference in response between the NRAO/CMU tipper and the SUMMIT instruments could arise from several different reasons: slight differences in the mesh filter (that could pick more or less flux from the strong atmospheric line at $837 \mathrm{GHz}$ ), in the black body constructions, in the window material, or in the optical characteristics.

Observed atmospheric opacity depends on the filter characteristics. At the zenith, Equation (6) holds

$$
1-\exp (-\tau)=\frac{\int_{0}^{\infty} \theta_{\text {filter }}(\nu) \cdot\left\{1-\exp \left[-t_{0}(\nu, \text { PWV })\right]\right\} \mathrm{d} \nu}{\int_{0}^{\infty} \theta_{\text {filter }}(\nu) \mathrm{d} \nu}
$$



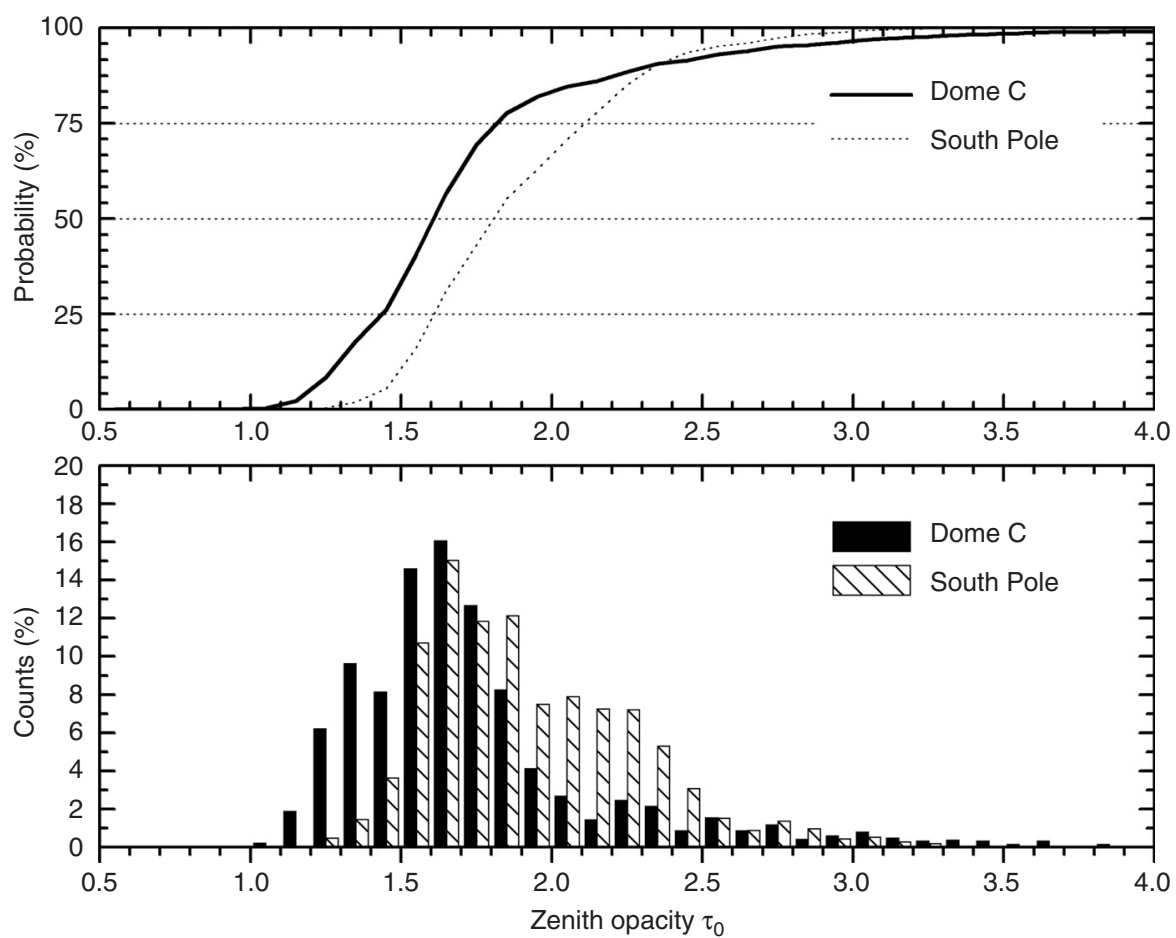

Figure 5 Histogram and probability distributions of the atmospheric opacity measured at Dome $\mathrm{C}$ and the South Pole between the 2000 December 8 and 2001 January 26, after binning data into $0.5 \mathrm{~h}$ intervals.

Table 2. Zenith broadband opacity at $350 \mu \mathrm{m}$ obtained at Dome $\mathrm{C}$ from SUMMIT and at the South Pole from NRAO/CMU tipper

Data taken 2000 December 21-2001 January 26. The offset between the two tippers, obtained from simultaneous observations at the South Pole had not been taken in account

\begin{tabular}{lcc}
\hline Quartile & Dome C & South Pole \\
\hline $25 \%$ & 1.43 & 1.61 \\
$50 \%$ & 1.61 & 1.80 \\
$75 \%$ & 1.81 & 2.11 \\
\hline
\end{tabular}

where $\tau_{0}$ is the derived sky opacity at the zenith for a given PWV content, $\theta_{\text {filter }}(\nu)$ the transmission of the filter at each frequency $\nu$, and $\tau_{0}(\nu, \mathrm{PWV})$ the atmospheric opacity at $v$ for a given PWV content.

To explore this further we calculated what opacity SUMMIT should measure for different values of $\tau_{0}$ and of the filter transmission function $\theta_{\text {filter }}(\nu)$ using the MODTRAN 4.0 simulation.

The results are shown in Figure 6. While a slight change in the central frequency does not significantly affect the instrument response, the opacity value at $860 \mathrm{GHz}$ is overestimated by an instrument with a wide bandwidth like SUMMIT. For opacity values around 1.7 (the Dome C summer campaign $25 \%$ quartile) the excess can reach 0.43 . Values obtained by a narrow-band instrument - for example the Fourier transform spectrometer (Pane et al. 2000) - would be lower by this amount with respect to values measured by one of the tippers.

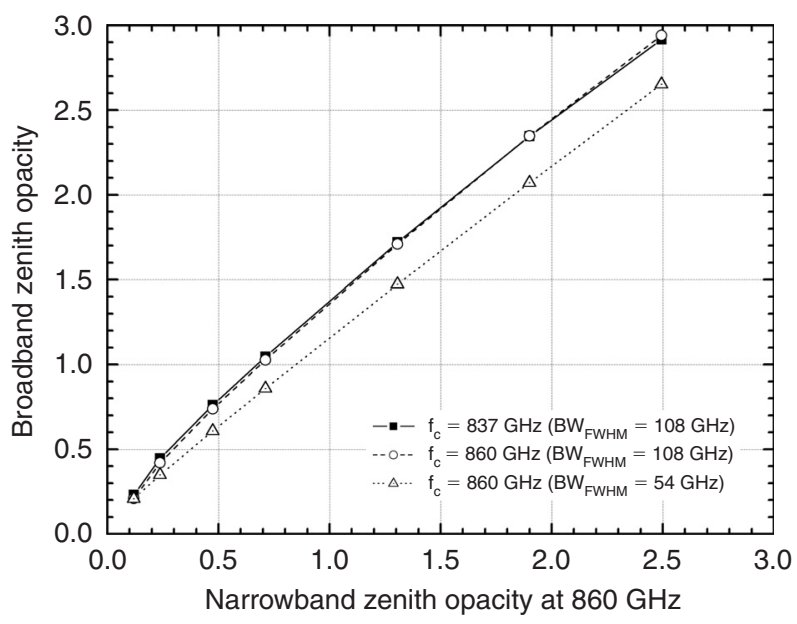

Figure 6 Calculated atmospheric opacity (see Equation (6)) as measured by a broadband radiometer as a function of the narrowband atmospheric opacity at $860 \mathrm{GHz}$. The continuous line shows the result expected from an instrument with a Gaussian bandpass filter with the same central frequency $f_{\mathrm{c}}$ and full-width half-maximum bandwidth $\mathrm{BW}_{\mathrm{FWHM}}$ as SUMMIT. The dashed line shows the expected results from a filter having the same bandwidth but centred on the transmission peak of the atmospheric window. The dotted line shows a filter centred on the window peak but with half the bandwidth.

\section{Conclusions}

SUMMIT has been shown to be a reliable, sensitive instrument for measuring the sky opacity and the effective atmospheric temperature at submillimetre wavelengths. During the five and a half weeks of operation at Dome C in summer 2000-2001 no malfunction was reported. 
While a preliminary direct comparison of summer data between Dome $\mathrm{C}$ and the South Pole is possible (although limited by the short period of observation), a meaningful comparison with data acquired at other latitudes (e.g. Mauna Kea and Llano de Chajnantor) is not straightforward as the effect of daily and seasonal variations dramatically changes with latitude. Further year-round observations are required to definitively compare the characteristics of the four sites. However, these preliminary data show that, as expected from straightforward morphological and geographical considerations, Dome C could represent one of the best sites in the world, if not the best accessible site, for submillimetre observations.

Although only five and a half weeks of data were obtained during this preliminary campaign, one might expect that mid-summer would be the time when conditions at Dome $\mathrm{C}$ would be least favourable with respect to the Pole, as Dome $\mathrm{C}$ is further north and the sun rises much higher in the sky. The next step is clearly to perform a year-round campaign to determine if Dome $\mathrm{C}$ is as good as these early measurements suggest.

\section{Acknowledgments}

This work has been supported by the Australian Research Council and by the Australian Antarctic Division. Sincere thanks to Ken Jackson, Pritipal Baweja, and Jack Sandall (UNSW) for carrying out, with great competence and in due time, all the modifications required to deliver the instrument. We thank the Italian Antarctic Program ENEA-PNRA and the French Antarctic Program IPEV for their invaluable support during the 2000-2001 summer Antarctic campaign at Dome C, and the American Antarctic Program for their support at the South Pole. Assistance from colleagues at the UNSW, especially Jon Everett and Jessica Dempsey, is gratefully acknowledged.

\section{References}

Afsar, M. N., Chi, H., \& Tkachov, I. I. 1995, in Millimeter and Submillimeter Waves II, Proc. SPIE, 73

Afsar, M. N., Tkachov, I. I., \& Wells, T. 1996, in Millimeter and Submillimeter Waves III, Proc. SPIE, 620
Ashley, M. C. B., Brooks, P. W., \& Lloyd, J. P. 1996, PASA, 13, 17 Burova, L. P., Gromov, V. D., Luk'yanchikova, N. I., \& Sholomitskii, G. B. 1986, SvAL, 12, 811

Calisse, P. G. 2004, PASA, 21, 252

Candidi, M., \& Lori, A. 2003, MmSAI, 74, 29

Chamberlin, R. A. 2001, JGR, 106, 20

Chamberlin, R. A., Lane, A. P., \& Stark, A. A. 1997, ApJ, 476, 428

Dopita, M. A., Ford, H. C., Bally, J., \& Bely, P. 1996, PASA, 13, 48

Dragovan, M., Stark, A. A., Pernic, R., \& Pomerantz, M. A. 1990, ApOpt, 29, 463

Godon, P., \& Cucinotta, N. 2000, in ISCORD2000 (Hobart, Australia)

Mastrantonio, G., Malvestuto, V., Argentini, S., Georgiadis, T., \& Viola, A. 1999, MetAP, 71, 127

Pajot, F., Gispert, R., Lamarre, J. M., Puget, J. L., Pomerantz, M. A., \& Peyturaux, R. 1989, in Astrophysics from Antarctica, eds. M. A. P. Dermott, J. Mullan, \& T. Stanev, Proc. AIP, 198

Pane, S., Blundell, R., Cosmo Papa, D., \& Barrett, J. W. 2000, PASP, 112,108

Radford, S. J. E. 2002, in Site Characterization for $\mathrm{mm} / \mathrm{submm}$ Astronomy, eds. Z. B. J. Vernin, \& C. Munoz-Tunon, ASP Conf. Ser., 148

Radford, S. J. E., \& Holdaway, M. A. 1998, in Advanced Technology MMW, Radio, and Terahertz Telescopes, ed. T. G. Phillips, Proc. SPIE, 486

Schwerdtfeger, W. 1984, Weather and Climate of the Antarctic (New York: Elsevier)

Smythe, W. D., \& Jackson, B. V. 1977, ApOpt, 16, 2041

Stark, A. A., et al. 2001, PASP, 113, 567

Storey, J. W. V. 1998, in Astrophysics in Antarctica: the AASTO program, eds. R. Landsberg, \& G. Novak (Chicago: ASP), 313

Storey, J. W. V., Ashley, M. C. B., Boccas, M., Phillips, M. A., \& Schinckel, A. E. T. 1999, PASP, 765

Storey, J. W. V., Ashley, M. C. B., \& Burton, M. G. 1996, PASA, 13,35

Storey, J. W. V., \& Hyland, A. R. 1992, ANARE Research Notes, 88,309

Storey, J. W. V., Ashley, M. C. B., Lawrence, J., \& Burton, M. G. 2003, MmSAI, Suppl. 2, 13

Townes, C. H., \& Melnick, G. 1990, PASP, 102, 357

Valenziano, L., \& Dall'Oglio, G. 1999, PASA, 16, 167 